\title{
EMPRENDIMIENTO VERDE EN TORNO A LA CONSERVACIÓN Y RECUPERACIÓN DEL AGUA Y SUS ESPACIOS EN BOGOTÁ Y MEDELLÍN (COLOMBIA)*
}

\author{
SONIA E. SANABRIA** \& ENRIQUE HURTADO**** \\ UNIVERSIDAD MILITAR NUEVA GRANADA
}

Recibido/ Received/ Recebido: 02/09/2016 - Aceptado/ Accepted / Aprovado: 03/10/2016

\begin{abstract}
Resumen
El emprendimiento verde es entendido en este documento, como el desarrollo de actividades orientadas a la mitigación, reducción o eliminación de problemáticas ambientales, las cuales son consideradas de entrada como innovadoras, en la medida en que proponen cambios en los patrones de producción y consumo que deterioran las condiciones naturales del planeta y en el relacionamiento del ser humano con el entorno. En este caso, el objeto de análisis es el emprendimiento verde orientado a la restauración y conservación del agua y sus espacios en Bogotá y Medellín. La investigación desarrollada se sustentó en la aplicación de entrevistas semi-estructuradas a actores de diferente tipo, involucrados en estas actividades. Se consideró que los espacios del agua son bienes comunes, alrededor de los cuales prevalece el sentir general de que pertenecen a todos y a la vez a nadie. Se concluye que un modelo eficaz para lograr su gestión adecuada implica la cogestión participativa, lo que abre la posibilidad al emprendimiento verde promulgado por diferentes actores. En este sentido, se encontraron emprendimientos promovidos por actores del sector público, empresas privadas, organizaciones sin ánimo de lucro, organizaciones sociales, fondos de agua, entre otros actores; algunos de los cuales intervienen directamente sobre los espacios del agua y otros indirectamente, a través de la optimización del consumo. La intervención en los espacios del agua en Bogotá y Medellín ha generado impactos positivos, no obstante, los esfuerzos aún son muy incipientes y de alcance local, teniendo en cuenta la extensión de los espacios del agua en el territorio.
\end{abstract}

Palabras clave: Agua; Espacios del agua; Bienes comunes; Emprendimiento verde.

Artículo de investigación producto del desarrollo del Proyecto ECO-1827 denominado "Caracterización del Emprendimiento Verde en Bogotá y Medellín alrededor de actividades orientadas a la conservación y restauración de los espacios del agua" financiado por la Vicerrectoría de Investigaciones de la UMNG para la vigencia 2015.

* Investigadora de la Facultad de Ciencias Económicas de la Universidad Militar Nueva Granada, Bogotá, Colombia. Contadora Pública y Magíster en Administración de la Universidad Nacional de Colombia. Grupo de Investigación CIE, categoría B en Colciencias, Universidad Militar Nueva Granada, Bogotá, Colombia. Correo electrónico: sesanabriaa@unal.edu.co

* Docente investigador de la Facultad de Ciencias Económicas de la Universidad Militar Nueva Granada, Bogotá, Colombia. Administrador de Empresas y Magíster en Administración de la Universidad Nacional de Colombia. Grupo de Investigación CIE, categoría B en Colciencias, Universidad Militar Nueva Granada, Bogotá, Colombia. Correo electrónico: enrique.hurtado@unimilitar.edu.co 


\title{
GREEN ENTREPRENEURSHIP AROUND THE CONSERVATION AND RECOVERY OF WATER AND ITS SPACES IN BOGOTÁ AND MEDELLÍN (COLOMBIA)
}

\begin{abstract}
Green entrepreneurship is understood in this document, as the development of activities aimed at mitigating, reducing or eliminating environmental problems, which are considered as innovative, insofar as they propose changes in production and consumption patterns that deteriorate the natural conditions of the planet and in the relationship of the human being with the environment. In this case, the object of analysis is the green enterprise oriented to the restoration and conservation of water and its spaces in Bogotá and Medellin. The research developed was based on the application of semi-structured interviews to actors of different types, involved in these activities. It was considered that water spaces are common goods, around which prevails the general feeling that they belong to all and at the same time to nobody. It is concluded that an effective model to achieve its proper management implies participatory co-management, which opens the possibility to green entrepreneurship promulgated by different actors. In this sense, there were found ventures promoted by actors of the public sector, private companies, non-profit organizations, social organizations, water funds, among other actors; some of which intervene directly on the water spaces and others indirectly, through the optimization of consumption. The intervention in the water spaces in Bogotá and Medellin has generated positive impacts, however, the efforts are still very incipient and of local scope, taking into account the extension of the water spaces in the territory. Keywords: Water; Water spaces; Common goods; Green entrepreneurship.
\end{abstract}

\section{EMPREENDIMENTO VERDE EM TORNO DA CONSERVAÇÃO E RECUPERAÇÃO DA ÁGUA E SEUS ESPAÇOS EM BOGOTÁ E MEDELLÍN (COLÔMBIA)}

\begin{abstract}
Resumo
O empreendimento verde é entendido neste documento como o desenvolvimento de atividades orientadas à mitigação, redução ou eliminação de problemáticas ambientais, as quais são consideradas primeiramente como inovadoras, na medida em que propõem mudanças nos padrões de produção e consumo que deterioram as condições naturais do planeta e no relacionamento do ser humano com o meio. Neste caso, o objeto de análise é o empreendimento verde orientado à restauração e conservação da água e seus espaços em Bogotá e Medellín. A pesquisa desenvolvida sustentou-se na aplicação de entrevistas semiestruturadas a agentes de diferente tipo, envolvidos nestas atividades. Considerou-se que os espaços da água são bens comuns, ao redor dos quais prevalece o sentir general de que pertencem a todos e ao mesmo tempo a ninguém. Conclui-se que um modelo eficaz para conseguir seu gerenciamento adequado implica a cogestão participativa, o que abre a possibilidade ao empreendimento verde promulgado por diferentes atores. Neste sentido, encontraram-se empreendimentos promovidos por agentes do setor público, empresas privadas, organizações sem ânimo de lucro, organizações sociais, fundos de água, entre outros agentes; alguns dos quais intervêm diretamente sobre os espaços da água e de outros indiretamente, através da otimização do consumo. A intervenção nos espaços da água em Bogotá e em Medellin tem gerado impactos positivos, não obstante, os esforços ainda são muito incipientes e de alcance local, tendo em conta a extensão dos espaços da água no território.
\end{abstract}

Palavras chave: Água; Espaços da água; Bens comuns; Empreendimento verde.

Sanabria, S. \& Hurtado, E. (2018) Emprendimiento verde en torno a la conservación y recuperación del agua y sus espacios en Bogotá y Medellín (Colombia). En: Revista de la Facultad de Ciencias Económica: Investigación y Reflexión. rev.fac.cienc.econ, XXVI (1), DOI: https:// doi.org/10.18359/rfce.3141

JEL: Q25, L26, L39. 


\section{Introducción}

Luego de décadas de recolección de evidencias científicas, hoy son indiscutibles las problemáticas ambientales derivadas de las prácticas depredadoras promovidas por el modelo económico contemporáneo y del modo de vida adoptado por la humanidad luego de la revolución industrial, con sus concepciones sobre éxito y calidad de vida, asociadas por las masas a capacidad de consumo de bienes y servicios (Uslu, Hancioglu \& Demir, 2015); sin tener en cuenta los residuos generados, el impacto ambiental y los recursos no renovables consumidos (Zoninsein, 2014). Un modelo en donde la naturaleza ha sido concebida como uno más de los factores de producción, sin deparar en su disponibilidad limitada, así como en la capacidad de carga y resiliencia del planeta, el cual ha sido llevado a un punto de saturación sin retorno (Tutu, 2014; citado en Vazquez-Brust, Smith \& Sarkis, 2014; Zahedi \& Otterpohl; 2015).

El costo asociado a éste modelo de "desarrollo" inequitativo, del cual las mayores beneficiadas han sido las naciones industrializadas, ha sido entre otros, la alteración de los ecosistemas naturales, la pérdida de biodiversidad, la contaminación del aire y los acuíferos, la proliferación de conflictos ambientales por la propiedad de los recursos naturales, la manipulación genética con consecuencias negativas para la humanidad, y la que ha sido reconocida como la falla de mercado más grande asociada a este sistema: "el cambio climático", entre otros (Allen \& Malin, 2008; Delgadillo \& Alburquerque, 2010; Sanabria \& Sabogal, 2011; Sanabria \& Hurtado, 2013; Stern, 2007).

Dentro de los múltiples elementos cuya disponibilidad se ha puesto en riesgo bajo el modelo económico imperante, se encuentra el agua, la cual se ve amenazada por circunstancias tales como una mayor demanda debido al crecimiento demográfico de la población, requerimiento de mayores cantidades para la producción de alimentos y de energía, menor disponibilidad debido a los efectos adversos del cambio climático y la presencia de fenómenos meteorológicos extremos como sequías $e$ inundaciones, peor calidad del recurso disponible por la contaminación industrial y doméstica, conflictos por el acceso a este elemento en cuencas compartidas (Ministerio de Agricultura, Alimentación y Medio Ambiente, s.f.) y competencia entre los diferentes tipos de demandas: urbana/rural, presente/futura, cantidad/calidad, regiones en competencia, y prioridades hídricas/otras prioridades sociales (Torres, 2014; UNESCO, 2006). Según la UNESCO (2006) las situaciones que afectan la disponibilidad de agua en el planeta se derivan de cambios políticos, mala gestión y anomalías climáticas.

En síntesis, las actividades humanas han modificado las características originales del agua, convirtiéndola en un recurso no renovable en términos de su calidad o pureza (Inegi-gdf, 2002; citado en Torres, 2014), lo que ha puesto en riesgo la vida de las diferentes especies vivas, incluida la especie humana, y principalmente la de aquellas poblaciones que carecen de infraestructuras y servicios esenciales o que viven en zonas expuestas (IPCC, 2014) tal como se ha experimentado en las diferentes temporadas de sequía e inundaciones en Colombia. Todo ello como manifestación de la crisis de una sociedad en donde ha primado el beneficio individual sobre el beneficio colectivo, sustentada en una lógica de acumulación de riqueza y en la dominación de la naturaleza (Agenda departamental mujeres por el agua, 2011).

Es así como el uso y manejo inadecuado de los recursos hídricos es considerado uno de los factores de mayor limitación para el desarrollo sostenible a nivel mundial (Torres, 2014); en donde la solución más allá de estar ligada a la disponibilidad de recursos financieros, está asociada a una gestión adecuada, a una coordinación y movilización de todos los actores en una cadena compleja (Torres, 2014). Es en esta línea, en donde las empresas como creación humana para la organización de la actividad económica, tienen una gran responsabilidad, dado que si bien han sido señaladas como co-reponsables directas de la actual crisis ambiental, contradictoriamente, son reconocidas como agentes clave en el proceso de transición hacia un modelo más sostenible.

Lo anterior debido a que se argumenta que las empresas cuentan con la capacidad para reinventarse 
y a través de su función innovadora, producir la siguiente revolución industrial (Hawken et al., 1999, Senge \& Carstedt, 2001, Braungart \& McDonough, 2002, citados en Cohen \& Winn, 2007; Izaidin \& Koe, 2012; López, 2012; McEwen 2013; Pacheco, Dean \& Payne, 2010), incidiendo en la transformación cultural y en la creación de nuevos estilos de vida (Iyigun, 2015; Ratti \& Chhibber, 2014; Uslu et al, 2015). Esta responsabilidad también se extiende al individuo y a todo tipo de organizaciones sociales y políticas como actores que interactúan con el entorno y lo modifican.

Teniendo en cuenta lo mencionado, el presente artículo, tiene por objetivo socializar los resultados obtenidos como producto del desarrollo de un proyecto de investigación que tuvo como fin caracterizar el emprendimiento verde alrededor de actividades de conservación y restauración del agua y sus espacios, en Bogotá y Medellín. Para ello se iniciará con un breve recorrido por la problemáticas del agua y sus espacios en entornos urbanos, el agua como bien común, el emprendimiento verde y su relación con las fallas de mercado. Posteriormente se hará un recuento de la metodología usada en el estudio y se presentará un análisis de los resultados por tipo de actor, buscando establecer el perfil del emprendedor verde, el tipo de actividades que desarrollan, las motivaciones y barreras existentes. Finalmente se comparten algunas conclusiones.

\section{Marco teórico}

\subsection{Los espacios del agua como bien común}

Cuando se hace una observación de las problemáticas existentes alrededor del agua en ciudades como Bogotá y Medellín, asociadas principalmente a contaminación y falta de apropiación social, la primera pregunta que cabe formularse al respecto es ¿de quién es la responsabilidad de la conservación y protección de los espacios del agua urbanos, esto es ríos, quebradas y humedales?

La respuesta parte de señalar que existe un gran debate sobre la concepción social, económica y política del agua (Torres, 2014). Así están quienes la conciben como patrimonio de la humanidad y por tan- to como un derecho humano universal (Paquerot, 2003, Gleick, 1999, Deléage, 2003, Maris, 2003, Petrella, 2001, 2003, Barlow \& Clarke, 2003, citados en Canelón, 2008); quienes la consideran una necesidad debido a que es esencial para la vida (Barlow \& Clarke, 2003, citado en Canelón, 2008) y quienes la representan como una mercancía (Zegarra, 2014; De Villiers, 2002, citado en Canelón, 2008) o bien económico que genera beneficios, tiene utilidad y valor de uso, por lo que está sujeta a negociación (Zegarra, 2014; De Villiers, 2002, citado en Canelón, 2008). Dentro de los beneficios que se atribuyen al agua se incluye el consumo directo de agua potable, saneamiento básico, actividades económicas como la agricultura, la pesca, la generación de energía, la minería; así como el uso recreacional y estético y los servicios ambientales y eco-sistémicos de la misma (Zegarra, 2014).

El agua también es clasificada como: bien común, bien público o bien privado, dependiendo de su naturaleza, su uso y los derechos de propiedad establecidos sobre ella (Torres, 2014; Zegarra, 2014). Así para Valdovinos (2011):

“ ... el agua como un recurso natural disponible en el medio ambiente se diferencia del servicio del agua, el cual incluye un conjunto de etapas que van desde la captación del recurso, pasando por su purificación, almacenamiento, distribución y saneamiento hasta su restitución al medio natural. El servicio del agua es un servicio público, ya que se trata de una actividad de interés general, o en otras palabras, de una actividad que trasciende los intereses particulares" (Valdovinos, 2011).

El derecho colombiano ha establecido el servicio de agua como de dominio público y por tanto su prestación se encuentra regulada por el Estado (Indij y Hantke, 2013), así mismo, ha considerado los ríos, lagos, humedales, y en general todas las formas del agua como bienes de uso público, con la sola excepción de las aguas que nacen y -mueren en una misma heredad (Agenda departamental de las mujeres por el agua, 2011). En relación con los espacios del agua 
en las áreas urbanas, y en ausencia de regulaciones efectivas, antes que ser considerados bienes públicos son bienes de uso común, alrededor de los cuales el sentir general es que son de todos y a la vez de nadie y que por tanto al ser compartidos entre muchos pueden ser objeto de desgaste (Torres, 2014). De acuerdo con Héritier, (2002, citado en Valdovinos, 2011) los bienes comunes pueden ser clasificados en tres categorías: (1) bienes públicos puros, caracterizados por su accesibilidad y no rivalidad; (2) bienes de propiedad común, definidos como aquellos que son accesibles a todos y su consumo por parte de un individuo conlleva a un menor consumo por parte de otro individuo; y (3) bienes club, caracterizados por su acceso limitado y un consumo parcialmente no rival (Héritier, 2002, citado en Valdovinos, 2011).

En este sentido, los espacios del agua podrían considerarse bienes de propiedad común, debido a que los bienes comunes comparten las características de no exclusividad y rivalidad en su consumo. No exclusión, debido a que no tienen limitantes en el acceso para ningún miembro de la sociedad una vez hayan sido producidos y rivalidad, dado que el acceso por parte de un miembro de la comunidad restringe el acceso a otros miembros en las condiciones iníciales. En este sentido, en ausencia de instituciones y/o regulaciones, cualquier miembro de la comunidad podría usar los cuerpos de agua urbanos para el vertimiento de desechos líquidos y sólidos, no obstante, estaría afectando su calidad y limitando a otros miembros de la comunidad para hacer uso de este recurso debido a la contaminación del flujo de agua, por lo que ya no podría ser disfrutado en usos recreativos y al ser el agua un recurso móvil, aguas abajo se estaría restringiendo su uso para actividades como el consumo, el riego o la ganadería (Zegarra, 2014). Este uso común incluye como actor al Estado, quien a través de la inadecuada gestión de las aguas servidas como resultado de la provisión del servicio público de agua, interfiere en el buen estado de los bienes comunes. Lo anterior podría asociarse a un tipo de una externalidad identificado por Zegarra (2014) en relación con los sistemas de extracción y distribución de aguas y es la relacionada con los efectos ecológicos.

Hardin (1968) quien es popularmente reconocido por su advertencia sobre la tragedia de los comu- nes señalaba que en el caso de la contaminación de los acuíferos, el problema no era sacar algo de los recursos comunes, sino ponerles algo dentro drenajes o desechos químicos, radioactivos o térmicos en el agua. Lo anterior, debido a que el ser humano en su racionalidad individualista, encuentra que su parte de los costos de los desperdicios que descarga en los recursos comunes es mucho menor que el costo de purificar sus desperdicios antes de deshacerse de ellos, lo cual conduce indefectiblemente a una degradación inevitable. Tal como lo sostenía Aristóteles "lo que es común para la mayoría es de hecho objeto del menor cuidado. Todo mundo piensa principalmente en sí mismo, raras veces en el interés común" (Ostrom, 2000, citada en Torres 2014). Frente a esta degradación de los bienes comunes Hardin (1968) proponía como solución leyes coercitivas o mecanismos fiscales que hicieren más barato para el contaminador el tratar sus desechos antes de deshacerse de ellos sin tratarlos.

Como solución general a la tragedia de los comunes, Hardin (1968) propuso el establecimiento de regímenes de derechos de propiedad sobre dichos bienes, a través de una solución de mercado: la privatización o de una solución de Estado: la gestión pública (Feeny, Berkes, McCay \& Acheson, 1990), sin embargo tiempo después se consideró que estos modelos eran ineficientes debido a que se centraban principalmente en la intervención de actores externos que imponen soluciones a los actores internos (Canelón, 2008), por lo que autores como Feeny, Berkes, McCay \& Acheson (1990) señalan que la evolución social ha demostrado la existencia de experiencias exitosas en donde la gestión comunal se ha convertido en una solución para detener dicha tragedia.

Por ello estos autores proponen como un esquema alternativo para la gestión de los bienes comunes: los derechos de propiedad comunal. Los autores parten de identificar cuatro categorías de derechos de propiedad, en cuyo seno los recursos de propiedad común son poseídos: acceso abierto, propiedad privada, propiedad comunal y propiedad estatal. Si bien sostienen que en la práctica algunos bienes son poseídos en formas coincidentes y algunas veces en combinaciones conflictivas de estos regímenes. 
Tabla 1. Derechos de propiedad sobre bienes comunes

\begin{tabular}{|l|l|}
\hline $\begin{array}{c}\text { Derechos de } \\
\text { propiedad }\end{array}$ & \multicolumn{1}{c|}{ Descripción } \\
\hline Acceso abierto & $\begin{array}{l}\text { Ausencia de derechos de propiedad, donde los bienes son gratuitos y abiertos a todos, lo que ha llevado al sentir general de } \\
\text { que los bienes comunes están expuestos al deterioro inevitablemente }\end{array}$ \\
\hline Propiedad privada & $\begin{array}{l}\text { Los derechos para emplear los recursos y excluir a otros son otorgados a un individuo o grupo de individuos. En ocasiones este } \\
\text { régimen no es eficiente debido a que puede generar altos costos y no se garantiza que sean respetados, adicionalmente puede } \\
\text { promover el uso insostenible del recurso y la sobreexplotación para beneficio de algunos (Bollier, 2008, citado en Gutiérrez, } \\
\text { 2011). }\end{array}$ \\
\hline Propiedad comunal & $\begin{array}{l}\text { Los derechos son poseídos por una comunidad reconocida de usuarios interdependientes, en donde al interior de la comunidad } \\
\text { es frecuente que los derechos de acceso y uso sean iguales, la regla es que este régimen suele ser más exitoso, sin embargo } \\
\text { las características sociales y políticas de los usuarios de los recursos y la forma en que ellos se relacionan con el sistema } \\
\text { político nacional afecta la habilidad de los grupos locales para organizar y gestionar la propiedad comunal (Ostrom, 1987, citada } \\
\text { en Cloquell, 2012). }\end{array}$ \\
\hline Propiedad Estatal & $\begin{array}{l}\text { Los derechos son concedidos exclusivamente al gobierno, el cual toma decisiones sobre el acceso, naturaleza y grado de } \\
\text { explotación, lo cual a veces es confundido socialmente como de acceso abierto, por lo que en ocasiones no son eficientes } \\
\text { para garantizar la exclusión. Cuando un bien pertenece al gobierno, el vínculo moral y legal con la ciudadanía empieza a diluirse } \\
\text { (Bollier, 2008, citado por Gutiérrez, 2011). }\end{array}$ \\
\hline
\end{tabular}

Fuente: elaboración de los autores, a partir de Feeny, Berkes, McCay \& Acheson (1990)

Feeny, Berkes, McCay \& Acheson (1990) en su revisión de la teoría de Hardin (1968) concluyen que esta teoría si bien fue perspicaz, es una teoría incompleta y no es determinística. Para estos autores no existe un único régimen de derechos de propiedad que sea efectivo en la gestión de los bienes comunes y sostienen que más bien depende de las complejas interacciones entre las características de los recursos, los derechos de propiedad y otros acuerdos institucionales, así como el entorno socio económico alrededor de cada recurso. Tanto la propiedad comunal, como la propiedad privada y la propiedad gubernamental han estado asociadas con éxitos y con fracasos. Ostrom (2000, citada en Cloquell, 2012) en este mismo sentido señala que el fracaso aparece en relación con la propiedad privada, la gubernamental y la común.

Feeny et al. (1990) también concluyen que en ocasiones la opción más viable es entonces compartir la regulación gubernamental o estatal con la autogestión de los usuarios. Esta co-gestión puede capitalizarse con base en el conocimiento local y el auto-beneficio en el largo plazo de los usuarios.

En América Latina se ha identificado la prevalencia de un esquema híbrido de gobernabilidad del agua en donde confluyen la existencia de múltiples formas institucionales, entre ellas: i) la gestión pública y descentralizada, ii) la privatización de los recursos con un lógica de mercado y la autogestión, lo que ha conllevado un aumento de movimiento sociales contra la privatización que conciben el agua como un derecho y no como una mercancía para el beneficio de unos pocos (Indij \& Hantke, 2013). Diferentes movimientos abogan por la gestión comunitaria, principalmente en aquellos lugares en donde el estado no hace presencia, teniendo en cuenta que los usuarios tienen gran conocimiento de la complejidad de la gestión, lo que permite un empoderamiento social y técnico de la comunidad. A su vez en lo que concierne a la gestión pública, propenden por el fortalecimiento de la participación y el control social con el fin de impulsar un modelo de gestión pública con mayor eficacia social, ambiental y económica reduciendo los casos de mala gestión (Delclòs i Ayats, 2008).

\subsection{Problemáticas alrededor de los espacios del agua en áreas urbanas de Bogotá y Medellín}

En general Colombia puede describirse como un país afortunado en lo que a disponibilidad de agua se refiere debido a su ubicación geográfica. Mira 
(2006) lo describe como uno de los 10 primeros países productores de agua (IDEAM, 2004, citado en Mira, 2006), el cual contaba a 2006 con 2.680.000 hectáreas de humedales, 743.000 cauces de aguas de $15.519 \mathrm{~km}$ de longitud fluvial; poseyendo 4 de las 214 grandes cuencas mundiales mayores a los $100.000 \mathrm{Km}^{2}$ (Magdalena, Caquetá, Guaviare y Meta) (Mira, 2006).

Lo anterior se convierte en una paradoja que ofende la dignidad del pueblo colombiano, cuando se estudia la provisión de agua a sus habitantes y se encuentra que en Colombia, casi 4 millones de habitantes no tienen agua potable y casi 7 millones carecen de saneamiento, lo cual equivale aproximadamente al $9 \%$ y al $15 \%$ de su población respectivamente (Indij \& Hantke, 2013). Según Indij \& Hantke (2013) ello se debe principalmente a los altos niveles de corrupción del gobierno, que impiden que los recursos que se destinan a la mitigación y erradicación de dichas problemáticas lleguen realmente a las poblaciones que los necesitan.

En este sentido de acuerdo con la voz de protesta de la Agenda departamental de las mujeres por el agua (2011):

“... el país del agua ha condenado a mucha de su gente a vivir en conflicto con el agua. Ante el despojo de sus tierras, la gente va a la orilla de ríos y cañadas y claro, joh, desastre natural! -Desastre natural- llaman al desastre social que ha construido una sociedad que hace del río cloaca, del humedal un pastizal, del páramo un sembradío de coca y amapola, condenando en este país del agua a la sed absurda de su gente, y todo por el lucro rápido y el enriquecimiento fácil" (Agenda departamental de las mujeres por el agua, 2011).

Lo anterior no se aleja de la situación descrita por Sabogal (2011) quien afirma que los espacios del agua $^{1}$ han sido ignorados en los procesos de expansión y urbanización de las ciudades, hasta el punto del desplazamiento de importantes fuentes de agua de sus lugares originarios, la extinción de algunos de ellos y el deterioro de los que aún existen. Los espacios del agua han sido contaminados, desviados, ocultados y/o canalizados, debido a que la percepción ciudadana y los valores que se han desarrollado alrededor de ellos, los reducen a un recurso útil para la supervivencia y la satisfacción de necesidades básicas, enmarcados en una visión netamente utilitarista, irrespetándosele su derecho a ser y a existir como elemento natural del paisaje. Los espacios del agua han sido incluso incorporados en el imaginario social como elementos negativos $e$ indeseables que solo han servido para el vertimiento de desperdicios (Sabogal, 2011). En el mismo sentido González (2014) expresa que los espacios del agua se ven totalmente transformados al superponer la geometría de la urbanización, en donde el agua se vuelve invisible.

Sumado a lo anterior, de acuerdo con Torres (2014) cuando han existido crisis ambientales, la acción pública no ha consistido en buscar medidas correctivas en relación con los factores generadores de la problemática, como la estabilización de la población y el crecimiento o desarrollo tecnológico o el ordenamiento territorial, sino más bien se ha enfocado en la ampliación de los sistemas de abastecimiento alcanzando fuentes más lejanas, provocando el trasvase de cuencas y trasladando el problema a otras áreas y a otras generaciones. Por lo tanto, de acuerdo con Mira (2006) la crisis del agua además de ser ambiental, es una crisis cultural.

Esta es exactamente la situación encontrada en los espacios del agua en ciudades como Bogotá y Medellín, en donde además de una tragedia de los comunes, hay una tragedia cultural y social y una ineficiencia en la gestión pública de los mismos, por lo que quizá el modelo más adecuado sea un modelo de co-gestión público, privado y comunitario. Y es que los sistemas de gestión del agua del siglo XXI

En este documento se entenderán como los espacios o el territorio que el agua ha usado tradicionalmente para desplazarse o concentrase, tales como quebradas, ríos y humedales 
no solo deben ser eficaces a fin de proporcionar esa garantía de suministro y así hacer efectiva la que se ha denominado "seguridad hídrica"; sino que además, deben ser sostenibles, de manera que permitan a los gobiernos y sus sociedades atender de manera eficaz y equilibrada los objetivos económicos, sociales y medioambientales asociados a todo modelo de crecimiento (Ministerio de Agricultura, Alimentación y Medio Ambiente, s.f.).

\subsection{El emprendimiento verde: una nueva pers- pectiva del emprendimiento}

Si bien la empresa y el emprendimiento han sido asociados históricamente a objetivos de generación de riqueza, incremento de la competitividad de los mercados globales y creación de empleo, la nueva ola de literatura sobre emprendimiento, ha añadido como otra de sus promesas, la responsabilidad social y la reducción del impacto ambiental de las actividades empresariales (Kuckertz \& Wagner, 2010; Silajdzic, Kurtagic \& Vucijak, 2015; Zahedi \& Otterpohl, 2015). Y es así, debido a que, si bien las empresas como creación humana para la organización de la actividad económica han sido señaladas como corresponsables directas de la actual crisis ambiental, contradictoriamente, son reconocidas como agentes clave en la transformación de la economía hacia un modelo más sostenible, con la capacidad para reinventarse y a través de su función innovadora, producir la siguiente revolución industrial (Hawken et al., 1999, Senge \& Carstedt, 2001, Braungart \& McDonough, 2002, citados en Cohen \& Winn, 2007; Izaidin \& Koe, 2012; López, 2012; McEwen 2013; Pacheco, Dean \& Payne, 2010). Lo anterior a partir de la innovación en gestión y tecnología; su rol en la transformación cultural y la creación de estilos de vida, inversión y gobernanza, introducción de nuevos modelos de negocio y nuevos enfoques organizacionales (Iyigun, 2015; Ratti \& Chhibber, 2014; Uslu et al., 2015).

En este sentido, la crisis ecológica actual es vista como una oportunidad para el emprendimiento en diversos sectores, tanto ambientales como tradicionales; y para la obtención de ventajas competitivas (Hamdouch, \& Depret, 2012) con la motivación intrínseca de modificar la dieta en la utilización de los recursos naturales y las prácticas relacionadas con la generación y gestión de desechos y emisiones de GEI (MAVDT, 2010; Farinelli et al., 2011). Bajo éste panorama, hay quienes afirman que ya ha surgido una nueva clase de emprendedores, denominados alternativamente como emprendedores sostenibles, emprendedores verdes o eco-emprendedores (Sanabria \& Hurtado, 2013), quienes transforman los métodos de producción convencionales, productos, estructuras de mercado y modelos de consumo, y los reemplazan con productos y servicios superiores ambientalmente a través de innovaciones ambientales (Hamdouch \& Depret, 2012).

Diferentes acepciones han sido introducidas en la literatura para referirse a esta nueva perspectiva del emprendimiento: emprendimientos de base ecológica (Delgadillo \& Alburquerque, 2010), ecoemprendimiento (OECD, 2011a), emprendimiento sostenible (Kuckertz \& Wagner; 2010), emprendimiento ecológico (OECD, 2011a) y emprendimiento sostenible y ambiental (Dean \& McMullen, 2007). Algunos autores usan varios términos de manera indiferente, como el caso de Gunawan (2014) quien usa emprendimiento verde y eco-emprendimiento como sinónimos, al igual que Silajdzic et al. (2015) y Nacu \& Avasilcăi (2014) quienes además usan los anteriores términos conjuntamente con la expresión emprendimiento ambiental.

Kuckertz \& Wagner (2010) se refieren al "emprendimiento sostenible" como aquel que además de satisfacer la definición de Schumpeter, gestiona el "triple bottom line" balanceando el bienestar económi$\mathrm{co}$, la equidad social y la resiliencia ambiental, esto es, es un emprendimiento que añade el beneficio de obtener más que un resultado meramente económico. Así mismo existen aportes que estudian el emprendimiento desde sus inclinaciones hacia una $\mathrm{u}$ otra dimensión de la sostenibilidad. Es así como se habla de emprendimiento social y emprendimiento ecológico, ambiental o como se le ha denominado en este texto, emprendimiento verde.

Gibbs (2009) usa el término eco-emprendedores y afirma que son visualizados como los agentes que conducirán el desarrollo de una nueva forma de capitalismo, a través de lo que se ha denominado 
la "modernización ecológica"; en dónde se buscan soluciones a problemáticas ambientales, poniendo el optimismo en el potencial del cambio tecnológico $e$ institucional.

Gunawan (2014) por su parte introduce cuatro categorías de emprendedores, que van desde el emprendedor regular cuyo principal fin son las ganancias en términos económicos; el emprendedor social que es aquel que realiza actividades innovadoras, que crean valor social y que puede ocurrir dentro o a través de organizaciones sin ánimo de lucro, comunidades, empresas, o sectores del gobierno (Tillmar, 2009, citado en Gunawan, 2014); el emprendedor verde o eco-emprendedor, que surge como una respuesta para tratar con el impacto ambiental negativo generado por la industria y que pueden ser clasificados en emprendedores sociales y emprendedores comerciales, en donde los primeros son aquellos que promueven productos, ideas y tecnologías eco-amigables a través o no del mercado y los últimos son personas que muestran su preocupación por el ambiente a través de la adopción de negocios eco-amigables (Pastakia, 1998, citado en Gunawan, 2014), estos últimos orientan sus negocios tanto ambientalmente como a la generación de ganancias; Y finalmente los emprendedores sostenibles que como se mencionó anteriormente observan los tres componentes de la sostenibilidad.

En síntesis, la crisis ecológica es vista también como una oportunidad para el emprendimiento en diversos sectores, tanto ambientales como tradicionales; y para la obtención de ventajas competitivas (Hamdouch, \& Depret, 2012). Para fines de este texto el emprendedor verde se entenderá desde la definición aportada por Gunawan (2014), según la cual este tipo de emprendedores pueden ser sociales o comerciales y pueden o no, promover sus productos, ideas o tecnologías a través del mercado.

\subsection{Emprendimiento verde alrededor de la ges- tión pública y comunal de un bien común}

Ahora bien, algunos aportes teóricos consideran el emprendimiento verde como una herramienta para contrarrestar fallas de mercado (Coase, 1974, Buchanan \& Faith, 1981, North \& Thomas, 1970,
Demsetz, 1970, Anderson \& Leal, 1997 y 2001 , citados en Dean \& McMullen, 2007) específicamente la falla asociada a los bienes comunes. En este sentido Dean y McMullen (2007) definen el emprendimiento sostenible y ambiental como el proceso de descubrir, evaluar y explotar oportunidades económicas que están presentes en fallas de mercado y conducirlas hacia actividades sostenibles potencialmente rentables relacionadas con el área ambiental.

En este sentido, en relación con los espacios del agua se puede identificar la existencia de la falla de mercado relacionada con la gestión de los bienes comunes, los cuales son de todos y a la vez de nadie, por lo que no existe un incentivo para su conservación. Adicionalmente se identifica la presencia de externalidades generadas por aquellos miembros de la comunidad quienes disponen sus vertimientos líquidos o sólidos en dichos espacios, sin considerar los problemas de contaminación que se generan. De conformidad con Dean \& McMullen (2007) este tipo de fallas de mercado generan oportunidades para el emprendimiento institucional a través de la promoción de regulaciones coercitivas que promuevan un cambio de comportamiento en los miembros de la comunidad y a su vez según Cohen \& Winn (2007), se genera la oportunidad de que diversos emprendedores desarrollen tecnologías y procedimientos para el tratamiento de vertimientos líquidos o sólidos antes de ser arrojados a las fuentes de agua, con lo cual se reversaría la externalidad existente.

\section{Metodología}

Los resultados que se sintetizan en este documento están sustentados en datos cualitativos recolectados a través de entrevistas semi-estructuradas, las cuales fueron aplicadas a un grupo de actores identificados a través de información secundaria obtenida previamente principalmente a través de internet, como agentes que intervienen en su entorno desarrollando actividades para la conservación y restauración de espacios del agua en Bogotá y Medellín.

Los actores entrevistados provienen de diferentes ámbitos sociales y económicos, incluyendo entida- 
des gubernamentales, empresas del sector privado, entidades sin ánimo de lucro y organizaciones sociales, a quienes se indagó principalmente por su perfil, el tipo de actividades que desarrollaban, la motivaciones que los condujeron a realizar la actividad orientada a la conservación y recuperación de espacios del agua, las dificultades y barreras que encontraban en el desarrollo de su labor y finalmente, si se consideraban o no como emprendedores verdes.

El instrumento aplicado en la presente investigación, de carácter semi- estructurado, conto con una serie de categorías y subcategorías como por ejemplo: financiación y si fue propia, externa o mixta; actores involucrados tales como comunidad, emprendedores, Estado; acciones verdes como conservación, reforestación, sensibilización, incidencia, articulación; innovaciones tales como, técnicas, tecnologías, redes de apoyo y su composición bien sea familiares, barriales, entidades públicas entre otras.

Se entrevistó un total de 17 actores, los cuales se clasifican como se muestra en la tabla 2.

\section{Tabla 2}

\begin{tabular}{|l|l|l|l|}
\hline \multirow{2}{*}{\multicolumn{1}{c|}{ Tipología }} & \multicolumn{2}{c|}{ Número de actores } & \\
\cline { 2 - 4 } & Bogotá & Medellín & Total \\
\hline Empresas del sector privado & 2 & 2 & 4 \\
\hline Entidades Públicas & 2 & 2 & 4 \\
\hline ONG's & 3 & 1 & 4 \\
\hline Organizaciones sociales & 1 & 1 & 2 \\
\hline Incubadoras de empresas & 0 & 1 & 1 \\
\hline Fondos del agua & 1 & 1 & 2 \\
\hline Total actores entrevistados & 9 & 8 & 17 \\
\hline
\end{tabular}

Fuente: elaboración de los autores

Para el análisis de las entrevistas se encontró oportuna su agrupación según la tipología de los actores, de manera que los resultados atendieran a un orden, buscando similitudes, diferencias e identificando una posición y/o contribución de cada categoría de actores a la conservación y restauración de los espacios del agua.
Dado lo heterogéneo de los grupos objeto de estudio se plantearon diferentes tipos de instrumentos para su aplicación, por lo que las entrevistas semi-estructuradas fueron una columna vertebral que pretendía obtener información acerca de las acciones verdes, los conceptos de emprendimiento verde, las diferentes motivaciones para emprender y las respectivas dificultades.

Desde lo público se inquirió sobre el papel del Estado en el desarrollo de las políticas pertinentes para el desarrollo y construcción de políticas ambientales. En emprendimiento que en la mayoría de los casos se traduce en la creación de empresa se indagó en la organización interna, el número de empleados, las actividades y el tamaño de la empresa entre otros.

\section{Resultados}

\subsection{Emprendedores alrededor de la conserva- ción y recuperación del agua en Bogotá y Medellín}

De acuerdo con Mira (2006) los movimientos sociales en torno al agua pueden clasificarse en tres categorías i) acciones coyunturales o movilizaciones populares en torno principalmente a los servicios públicos domiciliarios o megaproyectos; ii) proyectos de conservación o manejo de los recursos naturales, y iii) procesos de ordenamiento y apropiación del territorio. En concordancia con ésta clasificación, se puede afirmar que en Bogotá y Medellín se identificaron emprendimientos verdes que reflejan cada una de estas motivaciones y que incluso se han movido a través de ellas, encontrándose casos en donde la acción de los actores surgió motivada por una inconformidad en la prestación de un servicio público, pero que posteriormente evolucionaron a una motivación de conservación y manejo de los espacios del agua o de apropiación.

Por otro lado, al indagar sobre las actividades de emprendimiento verde que se desarrollan alrededor de los espacios del agua en Bogotá y Medellín, se evidencio la incidencia de diferentes tipos de actores, entre ellos, actores del sector público y privado, entidades sin ánimo de lucro, organizaciones socia- 
les formales e informales, fondos e incubadoras de empresas. Dentro de los actores que se incluyeron en el estudio, las entidades públicas, las ONG's y las empresas privadas representaron el mayor número, seguidas de las organizaciones sociales y los fondos y en mayor número las incubadoras de empresas de las cuales se incluyó solo una.

\subsubsection{Sector privado}

En relación con las empresas del sector privado incluidas en este análisis, se evidenció que si bien no desarrollan intervenciones directas sobre los espacios del agua, sus actividades innovadoras impactan indirectamente la calidad del recurso y las condiciones de dichos espacios. Lo anterior debido a que están orientadas principalmente al tratamiento de aguas servidas de carácter doméstico e industrial mediante el uso de tecnologías biológicas o químicas, que impiden que los vertimientos contaminados lleguen hasta los espacios del agua. Así mismo, contribuyen a la protección y conservación indirecta de estos espacios a través de acciones como la educación ambiental, la reforestación y el uso de aguas lluvias promoviendo la eficiencia en el consumo.

Las empresas incluidas en el análisis correspondieron principalmente a pequeñas empresas, relativamente nuevas en el mercado, pues no superaban los 15 años en desarrollo de sus actividades. Su alcance es principalmente local y nacional; y fueron fundadas principalmente por profesionales con estudios de postgrado, algunos de los cuales desempeñan profesiones asociadas al medio ambiente, quienes en algunos casos decidieron sumar esfuerzos con profesionales diestros en otras áreas para crear una oferta de servicios novedosa en un sector tradicional, como es el sector de la construcción.

Estos actores son principalmente emprendedores que respondieron a una oportunidad observada en el mercado, en algunos casos impulsada por la normatividad vigente. Manifestaron que además de tener un interés por contribuir a mitigar las problemáticas ambientales, los valores e historia individuales incidieron en la selección de la actividad alrededor de la cual desarrollaron su empresa. No obstante reconocen que la motivación económica tiene un mayor peso en la decisión de emprender frente a la motivación ambiental. Algunos actores asignaron una importancia de $35 \%$ a las motivaciones ambientales y de $65 \%$ a las motivaciones económicas.

En cuanto a las tendencias futuras de este tipo de iniciativas empresariales, según la perspectiva de los propios emprendedores, es que la actividad económica y el mercado tienden a expandirse en el futuro debido a que las problemáticas ambientales se están profundizando y así mismo los aspectos normativos tienden a ser cada vez más exigentes, demandando un cambio urgente en los patrones de producción y consumo actuales y por tanto generando mayores oportunidades para el emprendimiento verde innovador.

La mayoría de estos actores se identificaron con el concepto de emprendimiento verde y se definen como tal, debido a que consideran que con sus acciones contribuyen a mitigar los impactos negativos de la actividad humana en el ambiente. Uno de los actores estudiados resaltó la importancia de no considerar el emprendimiento verde como una moda temporal que sirve para entrar a mercados determinados, sino más bien como un acto consciente y responsable de la contribución que las empresas deben realizar al ambiente como agentes que interactúan en un entorno.

\subsubsection{Sector público}

Las entidades del sector público mayoritariamente de alcance local o nacional, a diferencia del sector privado, realizan intervenciones directas sobre los espacios del agua, pues como se mencionó en párrafos anteriores dichos espacios son considerados bienes comunes sobre los que se han establecido derechos de propiedad de carácter público. No obstante, la tendencia principal es que los actores del sector público no actúen de manera proactiva sino reactiva. Es decir que su accionar está motivado principalmente por la presión social que reclama su responsabilidad en torno a la recuperación de estos espacios, máximo cuando en ciudades como Bogotá y Medellín, el estado, es el responsable de la prestación del servicio de agua y alcantarillado $y$, por tanto, el responsable de gestionar el trata- 
miento de las aguas servidas. Esta presión social, ha dado ya algunos frutos, como acciones legales que le asignan responsabilidades concretas tanto al estado como a algunos actores privados; tal es el caso de la Sentencia del Río Bogotá (Consejo de Estado, 2014). Sin embargo, en términos generales la intervención del sector público ha estado mediada principalmente por la voluntad política de los gobiernos en curso como respuesta a la demanda social.

Como excepción a esta tendencia, se encontró el caso de una empresa pública de alcance internacional, cuyo estilo de gestión se asimila a la de una empresa privada, la cual considera el recurso hídrico como su activo estratégico, pues alrededor de él se teje su objeto social. Esta empresa actúa de manera proactiva, en donde el cuidado del recurso más que responder a un requerimiento social se fundamenta en la consciencia y la responsabilidad a lo largo de la cadena. Reconocen que si bien son una empresa pública no son una entidad de beneficencia y, por tanto, se reconocen como emprendedores verdes que buscan beneficios económicos a la vez que buscan ser pioneros en las actividades que desarrollan apuntándole a la sostenibilidad ambiental.

Las acciones de restauración y conservación de los espacios del agua promovidas por entidades del sector público, han buscado aplicar un enfoque integral $y$ participativo en donde se involucran diferentes actores incluyendo la comunidad circunvecina, organizaciones sociales y ONG's quienes a través de convenios son las encargadas de ejecutar acciones de compra de predios, estudios y diseños, adecuaciones hidrogeomorfólogicas, obras de restauración ecológica, apropiación social y educación ambiental, entre otras.

Dentro de los desafíos que enfrenta el sector público se encuentran la falta de continuidad de las políticas y las visiones promovidas por los diferentes gobiernos, el tiempo que conlleva realizar el proceso desde la compra del predio hasta las acciones de restauración, el cual suele ser de varios años, la cultura e idiosincrasia de la población en torno a los espacios del agua, la falta de apropiación social, falta de un ordenamiento territorial que proteja estos espacios, limitaciones de recursos.
En relación a la identificación de las entidades públicas con el concepto de emprendimiento verde, algunas entidades afirman serlo en la medida en que contribuyen a solventar problemáticas ambientales.

\subsubsection{ONG's}

El inicio de acciones alrededor de la restauración de los espacios del agua por parte de las ONG's incluidas en el estudio, data aproximadamente de hace 25 años como es el caso del actor 2 , lo cual es prueba fehaciente del inicio del movimiento social en torno a la defensa de estos espacios, incluso en una época en que este movimiento era considerado por algunos como algo exótico y romántico, de acuerdo a lo manifestado por las mismas ONG's. La motivación de los emprendedores verdes de esta categoría está relacionada con una consciencia temprana de las problemáticas ambientales, su preocupación por el futuro del agua en el planeta y el deseo de intervenir en la solución de problemáticas ambientales presenten en el territorio como contaminación, inadecuada gestión de desechos e inseguridad frente a lo cual decidieron organizarse y realizar una auto-gestión sobre tales situaciones. Algunas de estas ONG's fueron conformadas por personas con un sentido de pertenencia particular hacia el territorio, hacia el espacio del agua y con conocimiento de su historia como es el caso de los actores 1 y 3 .

Este tipo de organizaciones además de realizar actividades de carácter técnico ambiental en los espacios del agua a través de la recuperación, siembra, gestión social, etc. han buscado ganarse un espacio en las arenas políticas a través de la denuncia de problemáticas ambientales y la lucha por la expedición de normas que protejan dichos ecosistemas, además de velar por el cumplimiento de los instrumentos creados por la ley. Para ello buscan fortalecer sus redes con las comunidades y en algunos casos con otras organizaciones sociales a fin de consolidar el movimiento social.

Las ONG's estudiadas se financian principalmente con recursos propios, provenientes en algunos casos de capacitaciones organizadas por ellos mismos, voluntariado y principalmente de convenios 
realizados con instituciones del sector público para la restauración y conservación de los espacios del agua. Reconocen que su trabajo se ve continuamente amenazado por diversas dificultades, entre las cuales la más importante ha sido lograr un trabajo coordinado con las diferentes instituciones públicas que tienen responsabilidades frente a los espacios del agua, las cuales trabajan de manera desarticulada y parecen no tener claramente delimitadas sus funciones para atender a la ciudadanía.

\subsubsection{Organizaciones Sociales}

Como organizaciones sociales se clasificaron dos colectivos que no tienen identidad. Una de ellas data aproximadamente de hace 15 años (actor 1) y fue conformada por un grupo de profesionales de diversos campos del saber, como respuesta a problemáticas sociales evidenciadas en la comunidad en donde los actores estaban involucrados. Dicha organización no nació con el fin de respuesta a problemáticas ambientales, pero ligado a las problemáticas sociales que buscaban resolver fueron identificando que su alcance podría ser ampliado al campo ambiental en el área de intervención. Es así como empezaron a realizar intervenciones en espacios de agua como ríos y humedales, buscando integrar los saberes ancestrales de los indígenas muiscas, en donde se concibe la naturaleza como un espíritu y se busca una conexión mística con ella.

El actor 2 por su parte, es un colectivo organizado hace aproximadamente 7 años con el fin de atender una problemática social específica generada en torno al Río Arzobispo, en un punto específico de la ciudad, relacionada con los malos olores provenientes de las aguas servidas que circulan por allí. No obstante en el proceso, el colectivo se fue generando una apropiación en torno a las problemáticas de todo el río, hasta el punto de llegar a conformar un comité de cuenca.

Es así como este tipo de organizaciones realizan acciones como: limpieza de los espacios de agua, apropiación social a través de trabajo con la comunidad, siembra de árboles, etc. En el caso del actor 2 además ha buscado promover procesos de educación formal en relación con la gestión de ríos ur- banos como diplomados, también ha promovido el diálogo con las instituciones públicas, publicaciones, acciones de activismo ambiental, eventos culturales, proposición de proyectos para ser financiados por el gobierno distrital, entre otras.

No obstante, al igual que las ONG's manifiestan que su mayor dificultad es lograr una coordinación adecuada con las diferentes instituciones públicas que tienen responsabilidades frente a los espacios del agua. Manifiestan haber tenido disputas con personas que reclaman los predios recuperados ambientalmente y con entidades del Estado que manifiestan tener otros intereses en esos terrenos y tienen diferentes criterios sobre cómo se debe recuperar el espacio del agua. Adicionalmente cuando los espacios del agua son reconocidos legalmente y las entidades distritales asignan recursos para su recuperación y conservación, buscan introducir contratistas ajenos al territorio, ignorando el trabajo comunitario que ya se ha realizado por los colectivos, rompiendo el tejido social que se ha construido alrededor.

Otra de las dificultades de estos colectivos es que no tienen la personería jurídica para poder contratar y por tanto no pueden acceder a recursos públicos para financiar sus actividades, del mismo modo reconocen que las cuestiones ambientales no son una prioridad política en la ciudad y que falta un mayor involucramiento de la academia en la investigación de estas problemáticas.

Si bien estas organizaciones desean poder continuar con su trabajo, reconocen que el mayor logro fue haber comenzado, si bien consideran que no han realizado nada novedoso, puesto que se han servido de conocimientos existentes. Finalmente, en relación a la identificación de las organizaciones sociales con el concepto del emprendimiento verde, uno de los colectivos analizados, considera que su actividad no se puede enmarcar dentro de este término puesto que entiende el emprendimiento como un concepto atado al mercado y ellos no obtienen ningún tipo de ingreso de las actividades que realizan, por el contrario, invierten recursos. Más bien se consideran reparadores del daño ambiental que ha causado la sociedad al ambiente, sin que se denominen ambientalistas, puesto que creen que el ambientalismo es 
tan solo una moda y ellos mantienen una relación más estrecha con la naturaleza, desde el espíritu.

No obstante tal como se refirió en apartados anteriores, el emprendimiento verde se mueve entre las actividades ambientales puras y las actividades ambientales orientadas al mercado, por cuanto este tipo de actividades pueden enmarcarse en la definición adoptada para este documento.

\subsubsection{Fondos del agua}

Dentro del estudio llevado a cabo se incluyeron dos fondos del agua, el de Bogotá y el de Medellín respectivamente. Se identificó que este tipo de actores son más recientes, el más antiguo lleva 7 años en ejercicio de sus actividades, mientras el otro lleva tan sólo 2 años. Los fondos constituyen alianzas entre empresas públicas y privadas con el apoyo de The Nature Conservancy, cuyo fin es la protección del agua. Para ello las empresas que lo constituyen aportan unos recursos que son ejecutados por una organización independiente con conocimientos de temáticas ambientales. En el caso de Bogotá la organización ejecutora es una ONG dedicada a la conservación de los recursos naturales cuya existencia es independiente del fondo, en el caso de Medellín la organización ejecutora nació con el fin de administrar y ejecutar los recursos puestos a su disposición a través del mismo.

Los fondos de éstas dos ciudades tienen como fin conservar y proteger las fuentes abastecedoras de los acueductos que surten de agua la ciudad, y por tanto, están conformados tanto por las empresas encargadas de la provisión del líquido, como por empresas cuyo objeto social depende de la adecuada provisión del mismo, como lo son las empresas del sector de bebidas. Los recursos son invertidos en unas líneas y proyectos priorizados previamente. En el caso de Bogotá, los recursos son direccionados a la conservación de 245.000 hectáreas mediante el esquema de incentivos a la conservación, buscando generar cambios de comportamiento en la población ubicada en el área de influencia de las zonas de abastecimiento en relación con los recursos naturales, restauración a escala de paisaje, conectividad entre corredores, entre otras.
En Medellín los recursos del fondo se orientan a la conservación de 23.600 hectáreas de las cuencas abastecedoras del acueducto de Medellín y del Valle de Aburrá, con el fin de mejorar la calidad del agua. Los recursos se orientan al saneamiento básico y al manejo integral de cuencas, buscando involucrar a los diferentes actores desde el papel que cada uno tiene frente al recurso, así como en el rol de consumidor. A través del programa cultura del agua se realizan actividades de educación ambiental, comunicación y divulgación y se forman vigías del agua, también conocidos como "Guardacuencas". En el programa de gestión del recurso hídrico y la biodiversidad se trabaja predio a predio en la recuperación de los bosques de las riveras, nacimientos, humedales, mediante acuerdos de conservación con los propietarios. De igual forma, se implementan prácticas de producción sostenible en las fincas o buenas prácticas ambientales y finalmente, se propende por el mejoramiento de las prácticas productivas de las empresas asociadas al fondo.

Dentro de las limitaciones reconocidas por este tipo de organizaciones se encuentra la dificultad para desarrollar procesos de concientización en las personas, debido en parte a que las comunidades de las áreas de influencia de los acueductos, se sienten usadas y no sienten ninguna reciprocidad con la ciudad que toma sus recursos para su beneficio. Adicionalmente, debido a que en Colombia la conciencia popular es que existe abundancia de agua y no se reconoce la necesidad de trabajar por su conservación. Por tanto el mayor logro que consideran haber obtenido es el reconocimiento por parte de las comunidades y los lazos de confianza que se han creado en desarrollo de su labor, existiendo personas que incluso de manera voluntaria se acercan a éstas organizaciones para vincularse a las actividades que se desarrollan.

Al preguntarles si se consideraban emprendedores verdes reconocieron la existencia de diferentes actores interviniendo en los espacios del agua, por lo que lo innovador y lo emprendedor radica en la forma particular de hacer las cosas, que es lo que hace la diferencia. En el caso del fondo del agua de Bogotá consideran que en el momento en que fue creado fue totalmente innovador debido a que fueron pio- 
neros, no obstante, en la actualidad lo que los hace diferentes a los demás actores que interactúan en la misma área es la duración de sus procesos.

\subsubsection{Incubadoras de empresas verdes}

Otro tipo de actores involucrados en el estudio, fue una incubadora de empresas ambientales ubicada en Medellín, cuya labor viene desarrollando desde el año 2012, como repuesta a una iniciativa del gobierno nacional de apoyar el emprendimiento. Desde alli se realiza la evaluación a ideas o empresas con menos de 5 años de trayectoria, que se presenten y que den solución a una problemática ambiental previamente identificada en el Valle de Aburrá. Los emprendedores que se presentan tienen diferentes perfiles, desde estudiantes universitarios, personas que quieren dejar sus trabajos para emprender sus propios negocios, hasta agremiaciones sociales, etc. en donde se evidencia una mayor participación del género masculino.

La mayor parte de las iniciativas que se reciben están asociadas al sector de gestión de residuos, producción limpia y construcción sostenible. Si bien existe una línea de gestión del recurso hídrico, es en la que menos proyectos se presentan, el miembro de la incubadora que fue entrevistado sostiene que es debido a que en temas de tratamiento de aguas servidas se dispone de una planta de tratamiento de agua y en temas de acueducto la cobertura de EPM es amplia. Por otra parte, se reciben iniciativas sin visión empresarial que se deben más al amor por la naturaleza que no pueden ser apoyados, debido a que buscan es a una entidad que sostenga la fundación y el fin de la incubadora es realizar un acompañamiento de manera que se garantice la auto-sostenibilidad de dichos emprendimientos. En este sentido si bien, el fin último es solucionar una problemática ambiental, se debe tener una visión empresarial.

En lo que corresponde a la línea de gestión del recurso hídrico se ha hecho acompañamiento a emprendimientos de comercialización de trampas de grasa, trapeadoras que ahorran agua e iniciativas de turismo ambiental con componentes de educación bien establecidos. Hay otras iniciativas que no han sido factibles como potabilización de agua a partir de moringa, captación de agua lluvia, debido a que no tienen un mercado en lugares en donde existe la cobertura del servicio de acueducto por parte de la empresa de servicios públicos.

Dentro de las limitaciones que se han evidenciado, se destaca la existencia de mercados reducidos $e$ incluso la no existencia de un mercado para algunos productos, lo cual hace que así sean buenas ideas, no se pueden promover debido a que no hay quien los adquiera. De igual manera otra de las dificultades, principalmente de comercialización de productos a partir de productos reciclados es, que inicialmente los insumos no tienen costo, pero inmediatamente quien los genera se da cuenta que se está obteniendo un ingreso de su transformación, empieza a cobrar por ellos.

\subsection{Percepción sobre el concepto de emprendi- miento verde por parte de los actores}

Si bien se encontraron algunos actores que no se identificaron con el concepto de emprendimiento verde, la mayoría sí. El emprendimiento verde es entendido por los actores incluidos en el estudio, principalmente desde la perspectiva de los fines que persiguen las actividades que realizan. En este sentido la mayor parte de los actores al preguntárseles sobre si son o no emprendedores verdes, responden que sí, teniendo en cuenta que las actividades que realizan contribuyen de una forma $\mathrm{u}$ otra a la solución, mitigación, reducción o eliminación de una problemática ambiental.

Una visión que se aparta de esta tendencia fue el caso del actor 1 clasificado como organización social, quién manifestó tajantemente que "el emprendimiento es una palabra que viene del mercado, nos consideramos reparadores de los daños que ha hecho la sociedad, un emprendedor tiene que ser auto-sostenible, generar ingresos y vivir de eso. No tenemos una visión mercantilista de la naturaleza. No somos ambientalistas ni ambienta-listos. Nosotros somos Muiscas, gente de maíz, nos conectamos con los árboles, con las piedras. Ofrendamos en el rio Sumapaz todo lo que hacemos con la naturaleza" (Sic). 


\subsection{Motivaciones de los emprendedores verdes}

La literatura psicológica en el campo de la motivación permite comprender las diferentes clases de motivación que ocurren en la persona emprendedora, también permite comprender que factores intrínsecos y extrínsecos convierten a un individuo en empresario. Son muchos los factores que intervienen en el proceso de emprendimiento, desde los psicológicos hasta los económicos pasando por una serie de procesos emocionales y coyunturales que de alguna manera mueven a las personas a emprender actividades ligadas al medio ambiente.

En el caso particular de estudio, las motivaciones que llevaron a los actores al emprendimiento de actividades entorno a actividades de restauración, conservación y/o gestión del recurso hídrico varian dependiendo de la tipología de los actores. Así el sector privado es propositivo y generalmente busca aprovechar una oportunidad visualizada en el mercado, en algunos casos, las oportunidades estuvieron asociadas a la expedición de normatividad en este campo, relacionada por ejemplo con la gestión de vertimientos domésticos e industriales o para la recuperación de un espacio específico, como fue la sentencia para la recuperación del Río Bogotá (Concejo de Estado, 2014). A su vez, las empresas privadas no intervienen en los espacios del agua directamente sino en la fuente de la contaminación.

Por su parte, las entidades públicas son principalmente reactivas y responden a la presión social por la restauración y conservación de estos espacios, a excepción de una empresa pública que identificaba el agua como su activo estratégico y por ende el cuidado del mismo era algo completamente inherente a su actividad. Las organizaciones sociales respondieron principalmente a la solución de problemáticas de carácter local y al interés particular de los emprendedores por el tema ambiental.

\section{Discusión y conclusiones}

Las problemáticas que enfrentan los cuerpos de agua en la actualidad han venido consolidándose desde tiempo atrás, incluso desde el mismo momento en que un grupo de personas decidió asentarse permanentemente en el lugar que hoy ocupan las ciudades de Bogotá y Medellín. Estos espacios son bienes públicos que históricamente han sido dejados a la deriva, sin un doliente, convirtiéndose en cloacas de la urbanización y del desarrollo industrial, siendo ignorados e incluso detestados. Para empezar, estuvieron sujetos a cambios en su nombre desde los tradicionales nombres indígenas a nombres asignados en tiempo de la colonia, fueron canalizados perdiendo su escorrentía natural, siendo objeto de contaminación debido al vertimiento de aguas servidas en ellos. Por ende, en el imaginario social estos espacios empezaron a ser considerados caños o elementos negativos en el paisaje, puntos de depósito de desechos domésticos e industriales y zonas de invasión. En términos generales, los espacios del agua fueron ignorados en los procesos de planificación de las ciudades como espacios de conservación, conectores naturales y espacios de apropiación social.

Es necesario recordar que los espacios de agua de Bogotá forman parte del ecosistema de la llanura del rio Bogotá, la cual presta una variedad de servicios ambientales, lo mismo que funge como estación de aves migratorias y además regula las inundaciones que en la temporada de invierno genera el rio Bogotá.

Es innegable que ha sido la movilización social en torno a algunos espacios del agua la que ha empezado a demandar del Estado la asunción de la responsabilidad por su recuperación y el establecimiento de una autoridad para prevenir que los daños a estos espacios continúen creciendo exponencialmente. Es así como a través de diversos medios legales se han venido estableciendo responsabilidades al estado y al sector privado por el cuidado de estos espacios tal como lo sugiere la sentencia de recuperación del río Bogotá (Concejo de Estado, 2014) y diversas normas que regulan la disposición de residuos y vertimientos.

No obstante más allá de estas acciones legales, los actores incluidos en este estudio consideran que los espacios de planificación entre la ciudadanía y la autoridad local en donde los ciudadanos puedan expresar que es lo que quieren de estos espacios del 
agua, son incipientes. Por tanto generalmente las soluciones que se proponen desde lo público son impuestas, son soluciones temporales, soluciones atadas a un periodo de gobierno que no tienen continuidad y que están desarticuladas frente a las acciones que desarrollan otros actores en el territorio.

Otra de las alternativas que se evidenciaron a través de este trabajo es que si no existe cooperación desde el sector público, es la ciudadanía quien formula y desarrolla proyectos por cuenta propia, llegando al punto de establecer en algunos de estos espacios regímenes de uso y acceso, como es el caso de la quebrada las delicias, la cual ha sido consolidada ya como un destino turístico en los cerros de Bogotá. Con ello se ha demostrado que han sido las presiones comunitarias las medidas más efectivas para la gestión de estos bienes, y que la regulación es algo que ha seguido a la presión social.

En este sentido la movilización social y el marco normativo han abierto oportunidades al desarrollo de emprendimientos verdes en torno a la gestión del recurso hídrico en donde los actores dispuestos a pagar en el mercado por estos bienes y servicios hacen parte del sector púbico - quien debe asumir la responsabilidad de reversar el daño ya hecho - y actores del sector privado - quienes también deben realizar acciones de carácter proactivo para evitar seguir generando impactos a estos espacios del agua -; y es que la percepción de las personas es que la intervención en estos espacios corresponde al sector público.

Los emprendimientos provenientes de organizaciones sociales y ONG's, datan de hace un par de décadas, mientras los emprendimientos provenientes de empresas privadas, fondos e incubadoras son más recientes. Pues los primeros emprendimientos fueron los que generaron la presión social, que ha permitido el desarrollo normativo, para que el emprendimiento alrededor del agua surja como una oportunidad.

Por ahora los emprendimientos verdes en torno a estas actividades provenientes del sector privado han estado orientados a vender bienes y servicios a otros actores del sector privado a través de desa- rrollo e implementación de tecnologías para evitar el daño ambiental en los espacios del agua. Sin embargo la intervención directa sobre los espacios del agua a través de la limpieza, recolección de desechos vertidos en los mismos, reforestación, eliminación de asentamientos ilegales, conexiones erradas, etc. ha estado liderada por las comunidades y el Estado, en donde la intervención del sector privado ha sido mínima.

En relación directa a los espacios del agua, se concluye que aún existe un mercado que es reducido y poco atractivo para las empresas privadas y que se fortalece principalmente de la demanda social, la regulación y la voluntad política. Y ello es apenas evidente frente a un bien que es público y que a veces pareciere huérfano o sin doliente. Es así como este tipo de emprendimiento es apenas naciente, pero que se puede considerar en crecimiento, dadas decisiones jurídicas como la ya citada sentencia de descontaminación del Río Bogotá, en donde se obliga a los municipios ubicados a lo largo de él, al Distrito y las empresas privadas que vierten sus residuos en él, al desarrollo de actividades que contribuyan al mejoramiento de su calidad hídrica.

En concordancia con lo anterior, el mercado como institución primordial en el desarrollo económico debe encontrar a través del emprendimiento verde la creación de organizaciones dedicadas a la preservación y el mantenimiento de los espacios de agua en Bogotá y Medellín, como se ha hecho notar en la presente investigación con emprendedores locales que fungen como pioneros.

La mayor limitación manifestada por los actores incluidos en el estudio es la desarticulación de las entidades públicas locales y distritales, lo cual afecta negativamente tanto el estado de los espacios del agua como la actuación de los demás actores. Más aún teniendo en cuenta que los demás actores requieren de dichas entidades para poder actuar, lo anterior debido a que estos espacios son públicos y si las acciones se realizan de manera autónoma, posteriormente pueden presentarse conflictos de concepción. La mayoría de las ONG's y organizaciones sociales han sido motivadas por una preocupación específica sobre un espacio de agua por 
el cual tienen un sentimiento de identidad especial principalmente debido a que se encuentran ubicados en el lugar en donde estas personas habitan, con conocimiento especializado en el territorio y su historia.

Debemos incorporar los espacios de agua a la ciudadanía, por lo tanto la construcción de instituciones ambientales debe convertirse en prioridad, de tal manera que el valor del suelo desde el punto vista de terreno para construcción de vivienda pierda espacio ante la importancia ambiental para Bogotá y Medellín.

Queda como terreno fértil para posteriores investigaciones la manera como el Estado puede construir instituciones ambientales. Desde la disciplina económica, la ganadora del nobel 2009 E. Ostrom plantea como los derechos de propiedad común pueden ser reconocidos a una comunidad y de esta manera garantizar un mejor manejo a dichos bienes.

\section{Referencias}

Agenda departamental de las mujeres por el agua (2011). El agua un Derecho humano fundamental y un bien Público. Comité Departamental en defensa del agua y la Vida, Antioquia. Documento presentado al 7 Dialogo Interamericano del agua, Medellín.

Allen, J. \& Malin, S. (2008). Green entrepreneurship: a method for managing natural resources? Society and Natural Resources: An International Journal 21, (9).: 828-844

Canelón, J. (2008). Los bienes comunes: sentidos producidos sobre el agua del Valle de Quibor, Venezuela. Espacio Abierto Cuaderno Venezolano de Sociología, 17 (1): 109-142 ISSN 1315-0006 / Depósito legal pp 199202ZU44

Cloquell, M. (2012). Nuevo análisis "La tragedia de los comunes". Teoría y praxis. (11): 40-58.

Cohen, B. \& Winn, M.I. (2007). Market imperfections, opportunity and Sustainable Entrepreneurship. Journal of Business Venturing 22(1), 29-49.

Consejo de Estado (2014). Sentencia del 28 de marzo. Expediente A.P. 90479. Cuenca Hidrográfica del Río Bogotá.

Dean, T.J. \& McMullen, J.S. (2007). Toward a theory of sustainable entrepreneurship: Reducing environmental degradation through entrepreneurial action. Journal of Business Venturing, 22: 50-76.

Delclòs i Ayats, J. (2008). La gestión pública del agua con participación y control social. Hacia el derecho humano del agua. Viento sur, (98): 84-90
Delgadillo, J. \& Alburquerque, F. (2010). Emprendimientos de base ecológica, un modelo de interacción económica y territorial en Áreas Naturales y Protegidas de España y México. $1^{\mathrm{a}}$ ed., Instituto de Investigaciones Económicas-unam, Universidad de Sevilla, Colegio de Tlaxcala, México. 123 p.

Farinelli F., Bottini M., Akkoyunlu S. \& Aerni P. (2011). Green entrepreneurship: the missing link towards a greener economy. ATDF Journal, 8 (3/4): 42-48

Feeny, D. Berkes, F. McCay, B. \& Acheson, J. (1990). La tragedia de los comunes: 22 años más tarde. El jarocho verde. Human E Ecology, 18 (1). Traducción: Hipólito Rodríguez y Enrique Portilla Ochoa.

Gibbs, D. (2009). Sustainability Entrepreneurs, Ecopreneurs and the Development of a Sustainable Economy. Greener Management International Issue (55): 63-78. issn 0966-9671

González, C. (2014). Espacios del agua en el territorio urbanizado. Los Ângeles, California. Revista europea de investigación en arquitectura. REIA (2): 63-78. ISSN: 2340-9851

Gunawan, A. (2014). Preliminary Study of Classifying Indonesian Entrepreneurs. Procedia - Social and Behavioral Sciences 115: 243-250.

Gutiérrez, A. (2011). El grito de los bienes comunes: ¿qué son? Y ¿qué nos aportan? Rev. Ciencias Sociales 131-132: 127-145 / 2011 (I-II). ISSN: 0482-5276.

Hamdouch, A. \& Depret M-H. (2012). Green entrepreneurship networks and clusters: when the local requires the global. RSA global conference Junio. Beijing, China.

Hardin, G. (1968). The Tragedy of the Commons. New Series, 162 (3859): 1243-1248.

Indij, D. \& Hantke, M. (2013). Mapeo sobre integridad del agua en América Latina. Informe realizado en colaboración con Cap-Net y el Programa de Gobernabilidad del Agua del PNUD en SIWI.

IPCC (2014). Cambio climático 2014. Informe de síntesis. Contribución de los grupos de trabajo I, II y III al Quinto Informe de Evaluación el Grupo Intergubernamental de Expertos sobre Cambio Climático. Ginebra, Suiza, 157 págs.

Iyigun, N. (2015). What could Entrepreneurship do for Sustainable Development? A Corporate Social Responsibility-Based Approach. World Conference on Technology, Innovation and Entrepreneurship. Procedia-Social and Behavioral Sciences 195: 1226-1231.

Izaidin, A.M. \& Koe, W.L. (2012). Sustainable Entrepreneurship (SE): A Revised Model Based on Triple Bottom Line (TBL). International Journal of Academic Research in Business and Social Sciences, 2 (6): 293-310

Kuckertz, A. \& Wagner, M. (2010). The influence of sustainability orientation on entrepreneurial intentions - Investigating the role of business experience. Journal of Business Venturing 25: 524-539.

López, J. (2012). Modelos actitudinales y emprendimiento sostenible. Cuides. Cuaderno Interdisciplinar de Desarrollo Sostenible, Universidad de Almeria, (8): 111-132

McEwen, T. (2013). Ecopreneurship as a solution to environmental problems: implications for college level entrepreneurship education. International Journal of Academic Research in Business and Social Sciences. 3 (5): 264-288. 
Ministerio de Agricultura, Alimentación y Medio Ambiente (s.f.). Catálogo de gobernanza del agua en España. [citado 18 de julio de 2016]. Disponible en internet http://www.magrama. gob.es/es/agua/temas/sistema-espaniol-gestion-agua/

Ministerio de Ambiente, Vivienda y Desarrollo Territorial MAVDT. (2010). Política Nacional de Producción y Consumos Sostenibles. $72 \mathrm{p}$.

Mira, J. (2006). El agua un bien público. Gestión y Ambiente, Universidad Nacional de Colombia, Medellín, Colombia. 9(3):. 69-80.

Nacu, C. \& Avasilcăi, S. (2014). Technological Ecopreneurship: conceptual approaches. Procedia - Social and Behavioral Sciences 124: 229-235.

Organization for Economic Cooperation and Development. OECD (2011a). Entrepreneurship at a Glance 2011. [citado 06 de octubre de 2015]. Disponible en internet <http://dx.doi. org/10.1787/9789264097711-en>. 114 p.

Pacheco, D., Dean T. \& Payne, D. (2010). Escaping the Green Prison: Entrepreneurship and the Creation of Opportunities for Sustainable Development. Journal of Business Venturing, 25: 464-480.

Ratti, M. \& Chhibber, S. (2014). Green entrepreneurship: road to green economy-environment-sustainable social system. International Journal of Social Science \& Interdisciplinary Research IJSSIR, 3 (11): 82-95, ISSN 2277-3630. Disponible en indianresearchjournals.com.

Sabogal, C. (2011). Generar ciudad: recuperación del sistema hídrico dentro de la estructura urbana de Bogotá. Traza (4): 68-89. ISSN 2216-0647

Sanabria, S. \& Sabogal, J. (2011). Transferencia de tecnología en proyectos del mecanismo de desarrollo limpio (MDL) en Colombia. Sexto Encuentro de Investigaciones de la Universidad Militar Nueva Granada. Bogotá, 2011. 10 p
Silajdzic, I. Kurtagic, S. \& Vucijak, B. (2015) Green entrepreneurship in transition economies: a case study of Bosnia and Herzegovina. Journal of Cleaner Production 88: 376-384.

Stern, N. (2007). El informe Stern: La verdad sobre el cambio climático. Barcelona: Paidós.

Torres, L. (2014). Sistema Lerma: una visión política en la gestión pública del agua, ¿solución Estatal o Federal?. ISBN: 978607-8087-16-7.

UNESCO (2006). El agua una responsabilidad compartida, $2^{\circ}$ informe de las Naciones Unidas sobre el desarrollo de los recursos hídricos en el mundo. Capítulo 11. Compartir el agua. [citado 18 de julio de 2016]. Disponible en internet http://webworld.unesco.org/water/wwap/wwdr/wwdr2/ pdf/wwdr2_ch_11_es.pdf

Uslu, D., Hancioglu, Y. \& Demir, E. (2015). Applicability to Green Entrepreneurship in Turkey: A Situation Analysis. World Conference on Technology, Innovation and Entrepreneurship. Procedia-Social and Behavioral Sciences 195: 1238-1245.

Valdovinos, J. (2011). De la gestión local a la gobernanza global. Actores e interacciones multi-niveles en la gestión del agua en la ciudad de México. Regions \& Cohesión. Volume 1, Issue 3: 34-66.

Vazquez-Brust, D. Smith, A. \& Sarkis, J. (2014). Managing the transition to critical green growth: The 'Green Growth State. Futures 64: 38-50.

Zahedi, A. \& Otterpohl, R. (2015). Towards Sustainable Development by Creation of Green Social Entrepreneur's Communities. 12th Global Conference on Sustainable Manufacturing. Procedia CIRP 26: 196-201.

Zegarra, E. (2014). Economía del agua. Conceptos y aplicaciones para una mejor gestión. Grupo de Análisis para el Desarrollo (GRADE). ISBN: 978-9972-615-79-5.

Zoninsein, L. (2014). Economía Verde. Lima: Soluciones Prácticas. 63 p. Biblioteca Nacional del Perú N²014-17811 